\title{
Sexualidad en adolescentes escolarizados: Un análisis comparativo de género
}

Sexuality in adolescents: A Comparative gender analysis

Angelica de Jesús Valencia Valencia. ${ }^{1} \&$ Mayra Cristina Mena Mena. ${ }^{2}$

\begin{abstract}
DOI: https://doi.org/10.33262/concienciadigital.v4i4.1.1924

Introduction: Adolescence involves multiple processes of human development with biological and physiological changes that are psychologically complemented by sexuality. Objective: This research's main objective was to compare the knowledge regarding sexual education adolescents between female and male gender at Unidad Educative Santa Rosa. Method: a quantitative gender comparison was made in 193 First Year High School students. The comparison was made with the use of a survey composed of 15 questions which were divided into three main sections such as generalities and concepts of terms, namely gender, sex, sexual education as a human right; prevention of teenage pregnancy; and, other sexuality issues such as minority groups, and sexual harassment. The data obtained were consolidated and compared with a gender perspective. Results: the results obtained indicate a greater knowledge of sexuality by the female respondents; however, in general, there is little knowledge on these issues. Conclusions: after this process, it is concluded that there is a better knowledge of sexuality topics in the female population; however, there is an urgent need to propose an educational intervention with a sexuality approach with the students at Unidad Educative Santa Rosa.
\end{abstract}

Keywords: sexuality, gender, sex, teenage pregnancy, sexual harassment.

\footnotetext{
1 Pontificia Universidad Católica del Ecuador Sede Ambato, Dirección de Posgrado, Tungurahua, angelica.j.valencia.v@pucesa.edu.ec, ORCID: 0000-0003-1091-3367

2 Pontificia Universidad Católica del Ecuador Sede Ambato, Dirección de Posgrado, Tungurahua, cmena@pucesa.edu.ec, ORCID: 0000-0003-1091-3367
} 


\section{Resumen}

Introducción: La adolescencia conlleva múltiples procesos del desarrollo humano con cambios biológicos y fisiológicos que se complementan sicológicamente con la sexualidad. Objetivo: Esta investigación tuvo como objetivo principal el comparar el conocimiento referente a educación sexual en adolescentes escolarizados entre género femenino y masculino de la Unidad Educativa Santa Rosa. Método: se realizó una comparación cuantitativa de género en 193 estudiantes de Primer Año de Bachillerato. La comparación se realizó con la utilización de una encuesta compuesta de 15 preguntas las cuales estaban divididas en tres secciones principales tales como generalidades y conceptos de términos a saber género, sexo, educación sexual como derecho humano; prevención de embarazos en adolescentes; y, otros temas de sexualidad tales como grupos minoritarios, y acoso sexual. Los datos obtenidos fueron consolidados y comparados con enfoque de género. Resultados: los resultados obtenidos indican un mayor conocimiento de sexualidad por parte de las encuestadas de género femenino; sin embargo, en forma general, existe un escaso conocimiento sobre estos temas. Conclusiones: luego de este proceso, se concluye que existe un mejor conocimiento de temas de sexualidad en la población femenina; sin embargo, hay una necesidad urgente de plantear una intervención educativa con enfoque de sexualidad con los estudiantes de la Unidad Educativa Santa Rosa.

Palabras claves: Sexualidad, Género, Sexo, Prevención de embarazos, Acoso sexual

\section{Introducción}

La adolescencia es la última fase del desarrollo, la última etapa del individuo durante su curso hacia la madurez (Güemes-Hidalgo et al., 2017). Se le llama adolescencia al progreso evolutivo en la vida humana que comienza "biológicamente" con los cambios en la fisiología del pubis y completa "psicológicamente" con la organización última de la sexualidad (Soriano, 2015). Cubre el período comprendido entre la pubescencia, durante el cual aparecen las características sexuales secundarias y la edad de aproximadamente 18 a 20 años. El término "adolescente" no es del todo exacto: puede referirse a jóvenes de entre 10 y 18 años. Por tanto, es preferible considerar que el individuo pasa sucesivamente por etapas de la adolescencia (precoz, media y posterior) en lugar de clasificar a todos los adolescentes en la categoría de "adolescentes" (Güemes-Hidalgo et al., 2017).

Durante la adolescencia según Freud (1992), las zonas erógenas se resignan al dominio de la zona sexual, se plantean diferentes blancos sexuales para el hombre y la mujer y el individuo encuentra una pareja sexual más allá de los límites familiares. Por consiguiente, la adolescencia constituye un período de transición entre la organización del psiquismo infantil y el resultado de una personalidad adulta y madura. Durante esta fase evolutiva, el equilibrio psíquico se desestabiliza y la personalidad se caracteriza por la fluidez. El ego se vuelve vulnerable debido a la sucesión de acciones regresivas y progresivas como 
resultado de su esfuerzo por organizar las nuevas experiencias de prudencia. El ego se inunda con los impulsos de la pubescencia, ya que se ve obligado a formar la nueva imagen del cuerpo sexualmente maduro en la representación de sí mismo. Todo este progreso activa transformaciones psicológicas en cadena.

El adolescente constituye un "sujeto en formación". Este término tiene un doble significado. En primer lugar, declara que la adolescencia es un progreso evolutivo y constructivo: la formación del sujeto. En segundo lugar, cuando el adolescente habla con sinceridad, siempre se abre al lado poético del mundo y al habla: palabras, signos, seres humanos y objetos. La adolescencia es un período de desarrollo y si los conflictos mentales no se resuelven con éxito, pueden formar un tipo especial de psicopatología durante la edad adulta. La adolescencia se divide en tres etapas: primera (11-14), media (14-17), última (17-20) y cada una tiene su propio estándar.

Por otro lado, el tema de la educación sexual en las escuelas cruza la frontera entre la educación y la salud y plantea cuestiones políticas complejas entre los activistas sociales y de salud progresistas y los tradicionalistas morales (Dides, 2006) y, como tal, es de relevancia para un amplio público de académicos, formuladores de políticas y educadores (Güemes-Hidalgo et al., 2017). De hecho, hay pocas áreas del currículo escolar que hayan generado tanta controversia constante como la educación en sexualidad (Manzano y Herves, 2015). A menudo es el foco de un acalorado debate político y la atención de los medios ya que es un tema educativo que se analiza desde varias aristas (Wainerman et al., 2008).

En general, la lógica predominante detrás de la educación sexual en las escuelas a nivel mundial se deriva de una perspectiva tradicional de salud pública que se centra en la prevención de conductas de riesgo que conducen a infecciones de transmisión sexual, embarazos no deseados y violencia sexual (UNESCO, 2021). Si bien se ha llevado a cabo una gran cantidad de investigación sobre los posibles efectos preventivos de la educación sexual (Alvarado, 2013; Mosqueda, 2020; Von Sneidern et al., 2016; Matos, 2020), se ha prestado poca atención a una perspectiva positiva centrada en el bienestar y / o educación sanitaria crítica, incluida la identidad, la diversidad y los derechos humanos (Manzano y Herves, 2015).

Cavazotti (2021), enfatiza que la Declaración Universal de los Derechos Humanos toma como prioridad la educación sexual en niños y adolescentes para desarrollar la personalidad del ser humano de manera integral. En este contexto, se planteó el objetivo de explicar de qué manera tanto Colombia, como Chile y Ecuador han enfocado la educación sexual para verificar el cumplimiento o no de las leyes y preceptos internacionales. Luego de una revisión bibliográfica basada en los tratados de los derechos humanos, se concluye que estos países a pesar de las recomendaciones de las instancias internacionales, han abordado la sexualidad como un eje transversal en materia educativa. Además Muñoz (2017), pretendió realizar una revisión documental relacionada con la perspectiva de educación sexual en los campos de salud y educación en Latinoamérica. Después de realizar la búsqueda bibliográfica llegó a la conclusión que 
la educación sexual se aborda desde varias aristas tales como política, científica, religiosa, y demás. Esto trae consigo en la mayoría de casos, que se produzca una oposición entre las ideología o corrientes sociales, lo cual hace muy difícil un abordaje de este tema en el campo pedagógico. Por consiguiente, se puede manifestar que la tarea pedagógica se va endureciendo cada vez más en la historia de los países. La educación sexual dependerá de quienes estén en el poder de un país.

Por otro lado Baez (2015), revisó los principales abordajes de las políticas públicas referentes a educación de la sexualidad de jóvenes en Latinoamérica y el Caribe. Es así que, analizó los distintos enfoques que le dan los países a la educación de la sexualidad y los currículos de los mismos. Además, indagó lo referente a las tensiones que se crean en el camino de la inclusión de la perspectiva de género dentro de estos. De una forma puntual, analizó los conflictos creados desde la perspectiva feminista en pro de la justicia de género. La autora concluye, que en el campo de la educación sexual y la sexualidad de niños y jóvenes, en América Latina, todavía falta mucho por trabajar para la consecución de objetivos internacionales.

Por su parte Castro et al. (2016), enfatiza el gran problema que atraviesa el Ecuador, en tanto y en cuanto los jóvenes en edad estudiantil no tienen información relacionada con la sexualidad; por consiguiente, existen distorsiones en su orientación sexual. De esta manera, enfatizo la necesidad urgente de incorporar al currículo los temas de sexualidad que debería ser reforzado en el hogar. Tal como el caso de las investigaciones antes mencionadas a nivel de Latinoamérica, el caso del Ecuador no es una excepción, donde se debería llegar a un acuerdo para el beneficio de la comunidad y el buen ejercicio en temas de sexualidad y reproducción. De esta forma, manifiesta el autor, se contribuiría a disminuir los riesgos de contraer enfermedades venéreas que son producto de una indebida orientación.

Cabe recalcar, además, que en Ecuador existe un gran número de adolescentes que inician su vida sexual de manera prematura (Camacho y Jordán, 2018). Los autores analizaron las causas que provocan los embarazos prematuros no deseados. En el contexto de una investigación bibliográfica documental y tomando el criterio de estudiantes entrevistados, se indica que causa más sobresaliente para los embarazos no deseados es la pobre educación sexual en relación a métodos anticonceptivos. A decir de los estudiantes, sus padres deberían ser los encargados de educar a sus hijos en términos de sexualidad; pero, la escuela, también, deberían contribuir con una información global e integradora referente a la educación sexual para desarrollar un enfoque de respeto de los derechos humanos, la perspectiva de género y el desarrollo individual de los estudiantes quienes deberían dejar de verlo como un tabú.

En este sentido, esta investigación está ampliamente justificada por su vital importancia para la educación de los adolescentes. Una buena educación debe ir más allá del enfoque académico tradicional; también debe ayudar a los jóvenes a desarrollar el conocimiento, las habilidades y los valores éticos que necesitan para tomar decisiones conscientes y saludables sobre las relaciones, el sexo y la reproducción. 
El mundo está cambiando, muy rápidamente, con las tecnologías emergentes, la incertidumbre ambiental y la demografía global cambiante. Una educación de buena calidad es primordial. Es indispensable en un mundo marcado por la complejidad y la incertidumbre. Sin embargo, cuando hablamos de una buena educación, también debemos ir más allá del enfoque académico tradicional. Debemos buscar una educación que ayude a los jóvenes a desarrollar los conocimientos, las habilidades, los valores éticos y las actitudes que necesitan para tomar decisiones conscientes, saludables y respetuosas sobre las relaciones, el sexo y la reproducción.

Con base en la evidencia, sabemos que la educación integral en sexualidad debe ser una parte esencial de una buena educación que ayude a preparar a los jóvenes para tomar decisiones bien informadas en un mundo donde el VIH y el SIDA, las infecciones de transmisión sexual (ITS), los embarazos no deseados y la violencia de género sigue planteando graves riesgos para su bienestar.

Basado en datos brindados por el Departamento de Consejería Estudiantil (DECE), surge la necesidad de realizar una exploración de conocimientos a los estudiantes y tal como lo redacta la UNICEF en su revista Adolescencia una etapa fundamental. "La adolescencia es una de las fases de la vida más fascinantes y quizás más complejas, una época en que la gente joven asume nuevas responsabilidades y experimenta una nueva sensación de independencia" (UNICEF, 2002).

En virtud de lo expuesto, es necesario realizar una investigación que permita comparar la educación sexual en adolescentes escolarizados, generando referentes teóricos puesto para el desarrollo de futuras investigaciones sobre la temática, para lo cual se cuenta con acceso a la población participante, con la autorización de las autoridades de la institución, se dispone de los recursos y materiales necesarios y se aplicará un consentimiento informado a los padres para autorizar la participación de los adolescentes en la investigación. Es así, que surge la siguiente pregunta de investigación: ¿Cuál es la caracterización de conocimientos entorno a la educación sexual entre género femenino y masculino de adolescentes escolarizados de primer año de bachillerato?. En este contexto, la presente investigación pretende comparar el conocimiento referente a educación sexual en adolescentes escolarizados entre género femenino y masculino de la Unidad Educativa Santa Rosa.

\section{Metodología}

Se realizó una investigación cuantitativa, y alcance explicativo con el afán de recolectar información de los estudiantes sobre el nivel de conocimientos de sexualidad y realizar una comparación de género. Lo que permite una generalización de resultados y la toma de decisiones (Hernández et al., 2010). La investigación utilizó datos primarios obtenidos de los estudiantes mediante una encuesta. El diseño facilitó la recopilación de datos tanto cualitativos como cuantitativos en un intento de responder a la pregunta de la investigación. La variable independiente del estudio fue la educación sexual. Por otro lado, la variable dependiente fue el conocimiento de los estudiantes hacia la educación sexual. La población para este estudio estuvo constituida por 224 estudiantes 
matriculados en Primer Año de Bachillerato pertenecientes a las jornadas matutina como vespertina de las carreras de Contabilidad, Mecánica y Ciencias, de la Unidad Educativa Santa Rosa para el año académico 2021 - 2022; quienes están distribuidos como lo indica la tabla 1.

\section{Tabla 1}

Estudiantes de Primer Año de Bachillerato

\begin{tabular}{|c|c|c|c|c|c|c|c|}
\hline CURSO & ESPECIALIDAD & PARALELO & MASCULINO & $\%$ & FEMENINO & $\%$ & TOTAL \\
\hline $1 \mathrm{BGU}$ & Ciencias & A & 15 & $7 \%$ & 20 & $9 \%$ & 35 \\
\hline 1 BGU & Ciencias & B & 15 & $7 \%$ & 16 & $7 \%$ & 31 \\
\hline 1 BGU & Ciencias & $\mathrm{C}$ & 15 & $7 \%$ & 16 & $7 \%$ & 31 \\
\hline 1 BGU & Ciencias & D & 16 & $7 \%$ & 14 & $6 \%$ & 30 \\
\hline 1 BGU & Mecánica & A & 13 & $6 \%$ & 14 & $6 \%$ & 27 \\
\hline 1 BGU & Mecánica & B & 12 & $5 \%$ & 15 & $7 \%$ & 27 \\
\hline 1 BGU & Contabilidad & A & 10 & $4 \%$ & 13 & $6 \%$ & 23 \\
\hline \multirow[t]{2}{*}{$1 \mathrm{BGU}$} & Contabilidad & B & 12 & $5 \%$ & 8 & $4 \%$ & 20 \\
\hline & & TOTAL & 108 & $48 \%$ & 116 & $52 \%$ & 224 \\
\hline
\end{tabular}

Fuente: Unidad Educativa Santa Rosa (2021)

\section{Criterios de inclusión}

- Estudiantes debidamente matriculados y asistiendo a clases en las diferentes jornadas asignadas; así es; jornada matutina, estudiantes de las especialidades de Mecánica y contabilidad y jornada Vespertina, Especialidad de Ciencias en Primero de bachillerato de la Unidad Educativa Santa Rosa para el período académico 2021-2022.

- Estudiantes cuyos padres de familia y/o representantes legales firmaron su consentimiento informado para ser parte de este proyecto de investigación.

- Estudiantes que dispongan del tiempo necesario y la conexión a internet debida, fuera del horario de clases para realizar la encuesta

Los estudiantes seleccionados para esta investigación obedecen a los criterios de inclusión, arriba descritos; de tal manera que quedaron distribuidos como se muestra en la tabla 2.

Tabla 1

Población objeto de estudio

\begin{tabular}{lcc}
\hline Población & & \\
& Masculino & Femenino \\
\hline Frecuencia & 92 & 101 \\
Porcentaje & $48 \%$ & $52 \%$ \\
\hline \multicolumn{2}{l}{ Fuente: Unidad Educativa Santa Rosa (2021) }
\end{tabular}




\section{Tabla 2}

\begin{tabular}{lcc}
\multicolumn{3}{l}{ Estadística de edad de los encuestados } \\
\hline Edad \\
\hline $\mathrm{N}$ & Válidos & 193 \\
& Perdidos & 0
\end{tabular}

Promedio

16.25

Desviación Estándar

2.618

Fuente: Elaboración propia

Como se muestra en la tabla anterior, los encuestados tienen una edad promedio de 16.25 con una desviación estándar de 2.618.

Esta investigación tuvo como instrumento principal una encuesta sobre educación sexual la cual estaba dividida en tres secciones. La primera sección relacionada con generalidades de conceptos de sexualidad; la segunda parte, se centró en prevención de embarazos y la última parte a otros temas de sexualidad tales como grupos minoritarios, acoso sexual y demás. Esta prueba ha sido, además, validada por tres expertos en el ámbito de la Sicología Educativa.

Los estudiantes contestaron la encuesta, la misma que estuvo a su disposición en un Formato de Google de la cual se obtuvo una base de datos en Excel misma que se pudo homogeneizar para su posterior análisis. Para el análisis de la información se procedió a utilizar el software estadístico SPSS (Statical Product and Service Solutions).

\section{Marco Teórico}

Sexualidad.- La sexualidad implica todo un conglomerado de lo que el ser humano en su forma natural engloba a través de cada época de la vida. Incluye el comportamiento sexual, el género, la función fisiológica, valores, actitudes, el amor, las personas queridas, etc. Por ello es imprescindible, que se eduque en sexualidad desde muy tempranas edades, para tener plena conciencia y madurez en los temas de sexo. En este ámbito, los padres de familia deben conversar sobre sexualidad y relaciones dentro de una comunicación franca y natural para proporcionar información adecuada sobre respeto a sí mismos y a los demás, intimidad, comportamiento sexual conjugado con valores para que se sientan preparados para la vida (Camacho y Jordán, 2018).

Educación sexual como derecho de los niños niñas y adolescentes.- Los estándares internacionales de derechos humanos exigen que los gobiernos garanticen los derechos de los adolescentes a la salud, la vida, la educación y la no discriminación brindándoles educación integral en sexualidad en las escuelas primarias y secundarias, científicamente precisa y objetiva, libre de prejuicios y discriminación (Baez, 2015).

En virtud de los tratados internacionales de derechos humanos, los gobiernos están obligados a proteger y garantizar los derechos a la salud, la vida, la no discriminación, la 
educación y la información de sus poblaciones, en particular de los adolescentes (UNESCO, 2021). Estos derechos no pueden realizarse y disfrutarse plenamente si los adolescentes carecen de educación sexual (Manzano y Herves, 2015). Una comprensión integral de la salud sexual y reproductiva es imprescindible para la capacidad de un individuo para proteger su salud y tomar decisiones informadas sobre la sexualidad y la reproducción. De ello se desprende que dicha información es vital para reducir los embarazos adolescentes, embarazos y abortos inseguros, y prevención de la transmisión y propagación de infecciones de transmisión sexual (ITS) y VIH / SIDA entre los jóvenes (Calero et al., 2017).

Diferencias entre términos "sexo" y "género".- Para promulgar una eficiente educación sexual en el contexto educativo, se debe conceptualizar los términos "sexo" y "género", tomando en cuenta los Objetivos de Desarrollo Sostenible de la Organización de las Naciones Unidas, en especial el objetivo número 5 que hace referencia a la Igualdad de género (UNESCO, 2021).

Sexo y género son términos que a menudo se usan indistintamente, pero, de hecho, son dos conceptos diferentes, aunque para muchas personas su sexo y género son los mismos. El "sexo" se define como el conjunto de aspectos biológicos de un individuo según lo determinado por su anatomía, que es producida por sus cromosomas, hormonas y sus interacciones generalmente hombre o mujer algo que se asigna al nacer (Lamas, 2000). En cambio, el término "genero" se define como una construcción social relacionada con comportamientos y atributos basada en etiquetas de masculinidad y feminidad (Organización Mundial de la Salud [OMS], 2018); la identidad de género es una percepción personal e interna de uno mismo, por lo que la categoría de género con la que alguien se identifica puede no coincidir con el sexo que se le asignó al nacer donde un individuo puede verse a sí mismo como un hombre, una mujer, sin género o con un género no binario, donde las personas se identifican como en algún lugar del espectro entre el hombre y la mujer (UNESCO, 2021).

En síntesis, el sexo se basa en los conceptos de características definidas biológicamente, mientras que el género se basa en características construidas socialmente. Reconocen que existen variaciones en la forma en que las personas experimentan el género según la autopercepción y la expresión, y cómo se comportan. Esencialmente, casi todas las personas nacen con características físicas etiquetadas como masculinas o femeninas. En 1964, Robert Stoller acuñó el término identidad de género, que se refiere al concepto personal de un individuo sobre su género y cómo se siente por dentro (Hernández, 2006). Es un sentido interno de sí mismo profundamente arraigado y, por lo general, se identifica a sí mismo. La identidad de género difiere de la identidad sexual y no está relacionada con la orientación sexual de un individuo. Como tal, la categoría de género con la que se identifica una persona puede no coincidir con el sexo que se le asignó al nacer.

\section{Métodos anticonceptivos con enfoque que educación sexual}

Los anticonceptivos ayudan a proteger a las personas de quedar embarazadas y de contraer infecciones durante la actividad sexual. Es importante que todos los adolescentes 
aprendan sobre la anticoncepción para que tengan la información que necesitarán para tomar decisiones seguras y saludables. La Academia Estadounidense de Pediatría (AAP) recomienda que los padres hablen con sus adolescentes sobre cómo no tener relaciones sexuales es la mejor manera de prevenir las ITS, la infección por VIH y el embarazo. Sin embargo, estas discusiones también deben abordar opciones de anticoncepción confiables (Camacho y Jordán, 2018).

Los adolescentes pueden utilizar de forma segura cualquier método anticonceptivo. La edad no es una razón médica para negar cualquier método a los adolescentes. Las jóvenes suelen ser menos tolerantes a los efectos secundarios que las mujeres mayores. Sin embargo, con asesoramiento, sabrán qué esperar (Dides, 2006). Sin embargo, al tener más parejas sexuales, pueden enfrentar un mayor riesgo de contraer ITS (infecciones de transmisión sexual). Es importante al aconsejar a los jóvenes que consideren el riesgo de ITS y cómo reducirlo (Alvarado, 2013).

\section{Resultados}

Seguidamente se realizó el procesamiento de datos, lo cual, dio la pauta para obtener los siguientes resultados. En primer lugar, la encuesta tenía ocho preguntas dirigidas a los estudiantes de Primer año de bachillerato, relacionadas con las definiciones esenciales de educación sexual; como lo demuestra la tabla 4.

Tabla 3

Conocimiento general de definiciones

\begin{tabular}{|c|c|c|c|c|c|c|c|c|}
\hline \multirow[b]{2}{*}{ Frecuencia } & \multirow[b]{2}{*}{ Masculino } & \multicolumn{3}{|c|}{ Respuestas correctas } & \multicolumn{4}{|c|}{ Respuestas incorrectas } \\
\hline & & $\%$ & Femenino & $\%$ & Masculino & $\%$ & Femenino & $\%$ \\
\hline \multirow{2}{*}{$\begin{array}{l}\text { Generalidades } \\
\text { sexualidad }\end{array}$} & 81 & 88 & 79 & 78 & 11 & 12 & 22 & 22 \\
\hline & & $\%$ & & $\%$ & & $\%$ & & $\%$ \\
\hline \multirow{2}{*}{$\begin{array}{l}\text { Definición de la } \\
\text { palabra sexo }\end{array}$} & 72 & 78 & 79 & 78 & 20 & 22 & 22 & 22 \\
\hline & & $\%$ & & $\%$ & & $\%$ & & $\%$ \\
\hline \multirow{2}{*}{$\begin{array}{l}\text { Definición de la } \\
\text { palabra género }\end{array}$} & 35 & 38 & 56 & 55 & 57 & 62 & 45 & 45 \\
\hline & & $\%$ & & $\%$ & & $\%$ & & $\%$ \\
\hline \multirow{2}{*}{$\begin{array}{l}\text { Definición de la } \\
\text { palabra sexualidad }\end{array}$} & 80 & 87 & 85 & 84 & 12 & 13 & 16 & 16 \\
\hline & & $\%$ & & $\%$ & & $\%$ & & $\%$ \\
\hline \multirow{2}{*}{$\begin{array}{l}\text { Educación sexual } \\
\text { como derecho }\end{array}$} & 76 & 83 & 89 & 88 & 16 & 17 & 12 & 12 \\
\hline & & $\%$ & & $\%$ & & $\%$ & & $\%$ \\
\hline \multirow{2}{*}{$\begin{array}{l}\text { Definición del } \\
\text { término educación } \\
\text { sexual }\end{array}$} & 83 & 90 & 93 & 92 & 9 & 10 & 8 & $8 \%$ \\
\hline & & $\%$ & & $\%$ & & $\%$ & & \\
\hline \multirow{2}{*}{$\begin{array}{l}\text { Educación sexual } \\
\text { en el seno familiar }\end{array}$} & 88 & 96 & 97 & 96 & 4 & $4 \%$ & 4 & $4 \%$ \\
\hline & & $\%$ & & $\%$ & & & & \\
\hline \multirow{2}{*}{$\begin{array}{l}\text { Educación sexual } \\
\text { en la escuela }\end{array}$} & 10 & 11 & 16 & 16 & 82 & 89 & 85 & 84 \\
\hline & & $\%$ & & $\%$ & & $\%$ & & $\%$ \\
\hline \multirow[t]{2}{*}{ Total } & 92 & 100 & 101 & 100 & 92 & 100 & 101 & 100 \\
\hline & & $\%$ & & $\%$ & & $\%$ & & $\%$ \\
\hline
\end{tabular}

Fuente: elaboración propia

Esta información se refiere al conocimiento general de los términos sexualidad, sexo, 
género; así como también, la educación sexual como un derecho, dónde empieza y dónde debe ser reforzado a lo largo de la vida.

Los resultados obtenidos en la pregunta sobre generalidades de sexualidad indican que hay una tendencia positiva de conocimientos del concepto de sexualidad. No obstante, cabe recalcar que hay un $22 \%$ de estudiantes de género femenino que mostraron respuestas incorrectas frente a un $12 \%$ de estudiantes de género masculino. De igual manera, existe un porcentaje alentador con relación a la familiaridad demostrada con el término "sexo"; pero, se repite el mismo porcentaje de desaciertos en el género femenino. En la siguiente pregunta, relacionada con la definición de la palabra "género", 91 de 193 estudiantes, la saben; mientras que la mayoría, es decir 192 estudiantes no lo saben. Al respecto, la tendencia es que el $62 \%$ de la población masculina ha mostrado respuestas incorrectas frente a un $45 \%$ de la población femenina. Se debe analizar, también, que la gran mayoría de los estudiantes no tienen claro el concepto de la palabra "género". Por otro lado, la palabra "sexualidad" es desconocida para el 13\% de estudiantes de género masculino frente a un $16 \%$ de sexo femenino. Lo que evidencia, que, aunque sea bajo el porcentaje, las señoritas, muestran un mayor desconocimiento.

En el ámbito de la educación sexual como derecho de como estudiantes, la mayoría de estudiantes están conscientes de este derecho. No obstante, hay una diferencia de $17 \%$ de respuestas incorrectas del género masculino a un $12 \%$ del género femenino. Esto indica que las mujeres tienen más conciencia de su derecho en relación a educación sexual frente a los varones.

En lo referente a la frase "educación sexual", solo un 10\% de estudiantes de género masculino frente a un $8 \%$ de género femenino muestran respuestas incorrectas. Igualmente, se denota una ligera diferencia en el desconocimiento de los encuestados que tiende a ser muy baja con relación al deber de realizar educación sexual en el seno familiar. Por último, un $89 \%$ de estudiantes de género masculino en comparación con un $84 \%$ de encuestadas de género femenino, demuestran un desconocimiento del deber de las instituciones educativas en educación sexual. Es decir, la mayoría de encuestados no tienen conciencia de que la academia debe instruirlos en este tema.

La segunda parte de la encuesta se refiere a la prevención de embarazos como lo grafica de la tabla 5 .

\section{Tabla 4}

Conocimientos a cerca de prevención de embarazos en la adolescencia Prevención de embarazos en la adolescencia

\begin{tabular}{|c|c|c|c|c|c|c|c|c|}
\hline & Respuestas & orrec & & & Respuestas & ncorr & & \\
\hline & Masculino & $\%$ & Femenino & $\%$ & Masculino & $\%$ & Femenino & $\%$ \\
\hline $\begin{array}{ll}\text { Riesgo } & \text { de } \\
\text { embarazo } & \text { no } \\
\text { deseado } & \end{array}$ & 59 & $64 \%$ & 70 & $69 \%$ & 33 & $36 \%$ & 31 & $31 \%$ \\
\hline $\begin{array}{l}\text { Efectos sociales } \\
\text { del embarazo } \\
\text { no deseado }\end{array}$ & 28 & $30 \%$ & 58 & $57 \%$ & 64 & $70 \%$ & 43 & $43 \%$ \\
\hline
\end{tabular}




\section{Tabla 5}

Conocimientos a cerca de prevención de embarazos en la adolescencia (continuación)

\begin{tabular}{|c|c|c|c|c|c|c|c|c|}
\hline \multicolumn{9}{|c|}{ Prevención de embarazos en la adolescencia } \\
\hline & \multicolumn{4}{|c|}{ Respuestas correctas } & \multicolumn{4}{|c|}{ Respuestas incorrectas } \\
\hline & Masculino & $\%$ & Femenino & $\%$ & Masculino & $\%$ & Femenino & $\%$ \\
\hline $\begin{array}{l}\text { Píldora del día } \\
\text { después }\end{array}$ & 26 & $28 \%$ & 42 & $42 \%$ & 66 & $72 \%$ & 59 & $58 \%$ \\
\hline $\begin{array}{l}\text { Métodos } \\
\text { anticonceptivos } \\
\text { naturales }\end{array}$ & 43 & $47 \%$ & 50 & $50 \%$ & 49 & $53 \%$ & 41 & $41 \%$ \\
\hline Total & 92 & $100 \%$ & 101 & $100 \%$ & 92 & $100 \%$ & 101 & $\begin{array}{l}100 \\
\%\end{array}$ \\
\hline
\end{tabular}

Fuente: Elaboración propia

En la pregunta: de ninguna manera la adolescente puede quedar embarazada, el $36 \%$ de la población masculina frente a un $31 \%$ de la población femenina no responden acertadamente. Esto indica que más de la mitad de la población no saben cómo evitar el embarazo no deseado con tendencia ligeramente mayor a la población masculina. Por otra parte, con relación al conocimiento de los efectos sociales a consecuencia de embarazos en la adolescencia el $64 \%$ de los varones y el $43 \%$ de las mujeres, lo desconocen. Esto da una señal de alerta para proponer acciones inmediatas a nivel de la Unidad Educativa Santa Rosa. En lo relacionado con la ingesta y los efectos de la consabida "píldora del día después" el $72 \%$ de los encuestados de género masculino y el 58\% de la población femenina, lo desconocen. Al igual que en la pregunta anterior, vista la gran mayoría de los estudiantes con este desconocimiento, hace falta una intervención inmediata.

Por último, en lo relacionado a los métodos anticonceptivos naturales, la población masculina en un $53 \%$ lo desconocen en comparación con un $41 \%$ de la población femenina.

En general, en esta sección de la encuesta, aunque la población masculina demuestre mayor desconocimiento que la población femenina, como investigadora, lo considero alto. Por consiguiente, hay una evidencia de alerta en la comunidad educativa con la necesidad de brindar soporte a los estudiantes en estos temas que son trascendentales para su formación.

En la sección número tres de la encuesta se indagó sobre los conocimientos relacionados con temas de educación sexual tales como transexualidad, acoso sexual y educación sexual como derecho humano tal como lo refiere la siguiente tabla 6. 


\section{Tabla 6}

Otros temas de educación sexual

\begin{tabular}{|c|c|c|c|c|c|c|c|c|}
\hline \multicolumn{9}{|c|}{ Otros temas de educación sexual } \\
\hline & \multicolumn{4}{|c|}{ Respuestas correctas } & \multicolumn{4}{|c|}{ Respuestas incorrectas } \\
\hline & Masculino & $\%$ & Femenino & $\%$ & $\begin{array}{l}\text { Masculin } \\
\text { o }\end{array}$ & $\%$ & Femenino & $\%$ \\
\hline $\begin{array}{l}\text { Personas } \\
\text { transexuales }\end{array}$ & 32 & $35 \%$ & 48 & $48 \%$ & 60 & $65 \%$ & 53 & $52 \%$ \\
\hline Acoso Sexual & 31 & $34 \%$ & 61 & $60 \%$ & 61 & $66 \%$ & 40 & $40 \%$ \\
\hline $\begin{array}{l}\text { Educación sexual } \\
\text { integral como } \\
\text { derecho humano }\end{array}$ & 60 & $65 \%$ & 81 & $80 \%$ & 32 & $35 \%$ & 20 & $20 \%$ \\
\hline Total & 92 & $\begin{array}{l}100 \\
\%\end{array}$ & 101 & $100 \%$ & 92 & $\begin{array}{l}100 \\
\%\end{array}$ & 101 & $\begin{array}{l}100 \\
\%\end{array}$ \\
\hline
\end{tabular}

Fuente: elaboración propia

Estos temas de sexualidad, también han sido abordados arrojando los siguientes resultados. Preguntando a cerca de personas transexuales, los estudiantes en su mayoría no conocen sobre el tema ya que el $60 \%$ de la población masculina ha demostrado una respuesta incorrecta; así mismo las estudiantes en un 52\% han respondido erróneamente. Sin embargo, se denota una diferencia entre los porcentajes entre géneros dando un mayor número de respuestas erróneas al género masculino. En el ámbito relacionado con acoso sexual, el 66\% de los estudiantes de género masculino no conocen a cabalidad sobre el tema; igualmente, el $40 \%$ de las estudiantes desconocen sobre acoso sexual. Como los dos aspectos demuestran un alto porcentaje de respuestas erróneas, es menester, proponer una intervención educativa para que estos porcentajes bajen para beneficio de los jóvenes estudiantes. Finalmente, en lo referente a educación sexual como un derecho humano, el $32 \%$ de los estudiantes conjuntamente con el $20 \%$ de la población femenina no tienen plena conciencia de este hecho. Por lo tanto, este tema también es indispensable que sea socializado entre la población estudiantil.

Para resumir los resultados obtenidos en la encuesta con relación a género, se puede indicar que:

Tabla 7

Promedios finales comparación de género

\begin{tabular}{llll}
\hline Género & Media & $\mathrm{N}$ & $\begin{array}{l}\text { Desviación } \\
\text { Estándar }\end{array}$ \\
\hline Masculino & 8.74 & 92 & 2.489 \\
Femenino & 9.84 & 101 & 2.428 \\
Total & 9.32 & 193 & 2.512 \\
\hline
\end{tabular}

Fuente: Elaboración propia

Como se indica en la tabla 7 , se concluye que el conocimiento relacionado con 
sexualidad de los estudiantes de la Unidad Educativa Santa Rosa, son ligeramente diferentes dando una calificación más alta a las encuestadas de género femenino. Sin embargo, se debe recalcar que obtuvieron una calificación promedio de 9.32 sobre 15 , lo cual indica que los estudiantes en general necesitan una intervención inmediata.

\section{Discusión}

La educación en sexualidad impartida en un entorno de aprendizaje seguro y junto con el acceso adaptado a los jóvenes tiene un efecto positivo y duradero en la salud y el bienestar de los niños y los jóvenes. Es importante enfatizar que los niños y los jóvenes necesitan educación sexual formal e informal. Los dos no deben oponerse; se complementan entre sí. Si bien las escuelas son proveedores clave de educación formal en sexualidad, es fundamental que existan opciones para la educación en sexualidad no escolarizada, especialmente para llegar a los jóvenes más marginados y vulnerables, pero también como complemento de la educación en sexualidad escolar (Calero et al., 2017). Es así que la educación en sexualidad se ha convertido en una necesidad imperiosa en el mundo y por qué no decir en el Ecuador; debido a la existencia de patrones culturales y tabúes que hacen del convivir del adolescente un tema delicado (Figueroa, 2019).

Cabe también detallar que al realizar la búsqueda de antecedentes investigativos de esta índole que hayan sido propiciados en la Unidad Educativa Santa Rosa, no se encontró evidencia alguna. Al realizar la toma de la encuesta vía Google Forms, se mostró un cierto desinterés de los estudiantes por esta actividad. Los resultados descritos en la sección anterior dan cuenta de la poca información que existe en el plantel relacionado con el tema, siendo más acentuado en el tema de control de embarazos no deseados y la terminología de sexualidad abordada en este estudio.

Se considera que una de las causas para este problema sería la transversalidad de la educación sexual en la educación y que no haya una asignatura debidamente establecida en la malla curricular para tratar estos temas (Castro et al., 2016). Estos resultados son similares a los de Manzano y Herves (2015), quienes aseveran que educación sexual es una asignatura pendiente en el pensun académico de los jóvenes de educación media.

Teniendo en cuenta que las aulas son a menudo los únicos ámbitos en los que las discusiones sobre sexo o sexualidad se consideran temas escolares aceptables, y que el propósito de esta educación es facilitar el desarrollo saludable de todos los jóvenes, este artículo sugiere que incluir las cuestiones de sexualidad en la educación es muy importante. Esto sería llevado a cabo como un medio para apoyar el desarrollo de los estudiantes y mejorar sus experiencias de aprendizaje sin tabúes ni restricciones culturales. Derivado de una síntesis de la investigación descrita en este documento, las sugerencias proporcionadas tienen la intención de complementar este trabajo al abordar aspectos adicionales para el desarrollo de proyectos extra curriculares con contenido de educación sexual para facilitar un mejor conocimiento en los jóvenes de la Unidad Educativa Santa Rosa. En primer lugar, a pesar de la restricción causada por la Pandemia, se deberían desarrollar proyectos extra curriculares tendientes a resolver dudas de los 
estudiantes de una manera frontal y sana, lejos de personas que podrían dar información errónea a nuestros jóvenes. Por lo tanto, se sugieren las siguientes recomendaciones desde la perspectiva de proporcionar directrices a una institución educativa que desea mejorar la condición de conocimientos en educación sexual de sus estudiantes. Se recomienda que la institución educativa busque las estrategias para socializar temas relacionados con conceptos de educación sexual y prevención de embarazos en adolescentes que no se enfoquen en prejuicios no científicos y en supersticiones. Esto implica presentar información precisa y específica sobre diversos comportamientos sexuales, desafíos de salud, factores de protección y resiliencia de una manera que se ha demostrado que mejora el bienestar de los jóvenes y de grupos de minorías sexuales. Además, la elección de un proyecto institucional que brinde soporte educativo y emocional a los jóvenes.

Por lo tanto, se propone un proyecto institucional para palear de alguna manera la deficiencia en los conocimientos de los jóvenes relacionados con sexualidad, para futuras investigaciones donde toda la comunidad educativa esté integrada y trabaje mancomunadamente en temas de educación sexual para beneficio de los estudiantes.

\section{Conclusiones}

- La investigación aplicada arrojó datos importantes relacionados con el conocimiento referente a educación sexual en adolescentes escolarizados entre género femenino y masculino de la Unidad Educativa Santa Rosa. Dentro de este contexto se concluye que los estudiantes tanto del género masculino como del femenino necesitan una urgente intervención educativa; ya que los datos obtenidos evidencian deficiencia del conocimiento de los jóvenes de Primer Año de Bachillerato en el ámbito de la conceptualización de los términos sexo, género, educación sexual; además, de los temas relacionados con la prevención de embarazos en adolescentes y otros tópicos, tales como grupos minoritarios y acoso sexual.

\section{Referencias Bibliográficas}

Alvarado Thimeos, J. (2013). Educación sexual preventiva en adolescentes. Contextos, $N^{o} 29,2013,25-42$.

Baez Baez, J. (2015). Políticas educativas, jóvenes y sexualidades en América Latina y el Caribe. Las luchas feministas en la construcción de la agenda pública sobre educación sexual. Consejo Latinoamericano de Ciencias Sociales (CLACSO), 143.

Calero, E., Rodríguez, S., \& Aniocha, J. (2017). Abordaje de la sexualidad en la adolescencia. Cielo, 1.

Camacho, M., \& Jordán, J. (2018). La Educación Sexual: Como Método para la Prevención de Embarazos no Deseados en el Bachillerato. Revista de Investigación Enlace Universitario V(17), 49-55. 
Castro, A., Delgado, M., \& Pasos, A. (2016). La educación sexual en el Ecuador, una mirada crítica. Pacarina del Sur. Revista del Pensamiento Crítico Latinoamericano, 1-10.

Cavazotti Aires, D. (10 de Febrero de 2021). Chile, Colombia y Ecuador: educación sexual en convenciones internacionales e iniciativas estatales. Obtenido de Universidad Católica de Colombia: https://repository.ucatolica.edu.co/bitstream/10983/26051/1/Diogo\%20Cavazotti $\%$ 20Aires.pdf

Dides Castillo, C. (2006). Sexualidades y Modernidad. En C. Dides, Voces en Emergencia: EL Discurso Conservador y la Píldora Del Día Después (págs. 3957). Santiago de Chile: FLACSO.

Figueroa, P. (2019). Abordaje teórico en el estudio de las conductas sexuales de riesgo en la adolescencia. Rev Ciencias Médicas Scielo. Noviembre; 23(VI), 1-15.

Freud, A. (1992). El desarrollo adolescente. Barcelona: Paidós.

Güemes-Hidalgo, M., González-Fierro, M., \& Hidalgo, M. (2017). Desarrollo durante la adolescencia. Aspectos físicos, psicológicos y sociales. Pediatr Integral XXI (4), 233-244.

Hernández García, Y. (2006). Acerca del género como categoría analítica. Nómadas. Critical Journal of Social and Juridical Sciences, vol. 13, núm. 1, 1-11.

Hernández, R., Fernández, C., \& Baptista, M. (2010). Metodología de la Investigación. Mexico: The McGraw-Hill Companies, Inc.

UNICEF. (2002). Infancia, F. d. ADOLESCENCIA UNA ETAPA FUNDAMENTAL, 1.

Lamas Lamas, M. (2000). Diferencias de sexo, género y diferencia sexual. Cuicuilco. Vol. 7, núm. 18, enero-abril, 2000,, 1-24.

Manzano, D., \& Herves, E. (2015). Educación sexual: La asignatura pendiente. Maskana. Vol. 6 Núm. 1 (2015) , 27-38.

Matos Reyes, S. (2020). Estrategia educativa sobre las consecuencias de relación sexual precoz en adolescentes de la comunidad "El Carmen". Revista de Salud VIVE. Revista de Investigación en Salud. Volumen 3 | No. 8 | ISSN-L: 2664 - 3243, 85 94.

Mosqueda Padrón, L. (2020). Educación sexual: camino a la prevención del embarazo precoz. EduSol vol.20 no.72 Guantánamo jul.-set., 1-10.

Muñoz Astudillo, M. (2017). La educación sexual en latinoamérica: un campo de fuerzas en tensión. Cultura del Cuidado Enfermería. Vol. 14 N. 1, 57-70. 
Organización Mundial de la Salud [OMS]. (23 de Agosto de 2018). Género y salud. Obtenido de Organización Mundial de la Salud: https://www.who.int/es/newsroom/fact-sheets/detail/gender

Soriano Guillen, L. (2015). Pubertad normal y variantes de la normalidad. Pedriatr Integral XIX, 38-60.

UNESCO. (20 de Septiembre de 2021). Estudio mundial concluye que la educación sexual de amplio espectro es esencial para la igualdad de género y la salud reproductiva. Obtenido de UNESCO: https://es.unesco.org/news/estudiomundial-concluye-que-educacion-sexual-amplio-espectro-es-esencial-igualdadgenero-y

UNESCO. (2021). La UNESCO y la Declaración Universal de Derechos Humanos. Obtenido de https://es.unesco.org/udhr

Von Sneidern, E., Quijano, L., Paredes, M., \& Obando, E. (2016). Estrategias Educativas para la Prevención de Enfermedades de Transmisión Sexual en Adolescentes. Rev.Medica.Sanitas. Volumen 19 • No. 4 • 198-207.

Wainerman, C., De Virgilio, M., \& Chami, N. (2008). La Escuela y la Educación sexual. Buenos Aires: UNFPA. 


\section{PARA CITAR EL ARTÍCULO INDEXADO.}

Valencia Valencia, A. de J., \& Mena Mena, M. C. (2021). Sexualidad en adolescentes escolarizados: Un análisis comparativo de género . ConcienciaDigital, 4(4.1), 50-66. https://doi.org/10.33262/concienciadigital.v4i4.1.1924

\section{LCiencia}

El artículo que se publica es de exclusiva responsabilidad de los autores y no necesariamente reflejan el pensamiento de la Revista Conciencia Digital.

El artículo queda en propiedad de la revista y, por tanto, su publicación parcial y/o total en otro medio tiene que ser autorizado por el director de la Revista Conciencia Digital.

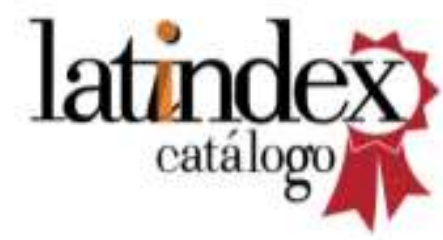

